\title{
Erratum to: Complement of forms
}

\author{
Zoltán Sebestyén · Tamás Titkos
}

Published online: 6 July 2013

(C) Springer Basel 2013

\section{Erratum to: Positivity (2013) 17:1-15 DOI 10.1007/s11117-011-0138-4}

The original publication of this paper contained an error in Lemma 2(g). The statement (which is used later in Theorem 6, Lemma 12, and Theorem 11) is false in general.

We will show that $\mathbf{D}_{\mathfrak{w}_{k}} \mathfrak{t} \uparrow \mathbf{D}_{\mathfrak{w}} \mathfrak{t}$ whenever $\mathfrak{w}_{k} \uparrow \mathfrak{w}$ and $\mathfrak{w}$ is dominated by $\mathfrak{t}$. Since this condition is satisfied in Theorem 6, Lemma 12, and Theorem 11, the results remain true (with the same proof).

The first part of the following theorem is the reformulation of (Proposition 5.1 in [1]) for nonnegative sesquilinear forms, the second part is an easy consequence. This is the correct version of Lemma $2(\mathrm{~g})$.

Theorem 1 Let $\mathfrak{t}, \mathfrak{w}$, and $\mathfrak{w}_{k}(k \in \mathbb{N})$ be nonnegative sesquilinear forms on the complex linear space $\mathfrak{D}$. Assume further that $\mathfrak{w}_{k} \uparrow \mathfrak{w}$, and $\mathfrak{w} \leq \alpha \mathfrak{t}$ for some $\alpha>0$. Then

$$
\sup _{k \in \mathbb{N}}\left(\mathfrak{w}_{k}: \mathfrak{t}\right)=\left(\sup _{k \in \mathbb{N}} \mathfrak{w}_{k}\right): \mathfrak{t}=\mathfrak{w}: \mathfrak{t} \text { and } \sup _{k \in \mathbb{N}} \mathbf{D}_{\mathfrak{w}_{k}} \mathfrak{t}=\mathbf{D}_{k \in \mathbb{N}} \mathfrak{w}_{k} \mathfrak{t}=\mathbf{D}_{\mathfrak{w}} \mathfrak{t} .
$$

Proof Since $\mathfrak{w}_{k}: \mathfrak{t} \leq \mathfrak{w}: \mathfrak{t}$ for all $k \in \mathbb{N}$, the supremum (denoted by $\mathfrak{s}$ )

The online version of the original article can be found under doi:10.1007/s11117-011-0138-4.

Z. Sebestyén · T. Titkos $(\varangle)$

Department of Applied Analysis and Computational Mathematics,

Eötvös Loránd University, Pázmány Péter sétány 1/c., Budapest 1117, Hungary

e-mail: titkos@cs.elte.hu

Z. Sebestyén

e-mail: sebesty@cs.elte.hu 


$$
\mathfrak{s}:=\sup _{k \in \mathbb{N}}\left(\mathfrak{w}_{k}: \mathfrak{t}\right)
$$

exists. We will show that $\mathfrak{s}=\mathfrak{w}: \mathfrak{t}$. Since

$$
\mathfrak{s}=\sup _{k \in \mathbb{N}}\left(\mathfrak{w}_{k}: \mathfrak{t}\right) \leq \mathfrak{w}: \mathfrak{t} \leq(\alpha \mathfrak{t}): \mathfrak{t}=\frac{\alpha}{\alpha+1} \mathfrak{t}
$$

one can apply (Theorem 3.4 in [3]). I.e., the parallel difference $\mathfrak{s} \div \mathfrak{t}$ exists and

$$
\mathfrak{t}:(\mathfrak{s} \div \mathfrak{t})=\mathfrak{s}=\sup _{k \in \mathbb{N}}\left(\mathfrak{w}_{k}: \mathfrak{t}\right) \geq \mathfrak{w}_{k}: \mathfrak{t} \quad(\forall k \in \mathbb{N})
$$

Hence, according to (Proposition 2.7 in [2]) one obtain that

$$
\mathbf{D}_{\mathfrak{t}}(\mathfrak{s} \div \mathfrak{t}) \geq \mathbf{D}_{\mathfrak{t}} \mathfrak{w}_{k} \quad(\forall k \in \mathbb{N}) .
$$

Since $\mathfrak{w}_{k}$ is almost dominated by $\mathfrak{t}$, it follows also that

$$
\mathfrak{s} \div \mathfrak{t} \geq \mathbf{D}_{\mathfrak{t}}(\mathfrak{s} \div \mathfrak{t}) \geq \mathbf{D}_{\mathfrak{t}} \mathfrak{w}_{k}=\mathfrak{w}_{k} \quad(\forall k \in \mathbb{N})
$$

Taking the supremum in $k, \mathfrak{s} \div \mathfrak{t} \geq \mathfrak{w}$ and

$$
\mathfrak{s}=(\mathfrak{s} \div \mathfrak{t}): \mathfrak{t} \geq \mathfrak{w}: \mathfrak{t} \geq \sup _{k \in \mathbb{N}}\left(\mathfrak{w}_{k}: \mathfrak{t}\right)=\mathfrak{s}
$$

Consequently,

$$
\sup _{k \in \mathbb{N}}\left(\mathfrak{w}_{k}: \mathfrak{t}\right)=\left(\sup _{n \in \mathbb{N}} \mathfrak{w}_{k}\right): \mathfrak{t}=\mathfrak{w}: \mathfrak{t} .
$$

It remains only to show that $\sup _{k \in \mathbb{N}} \mathbf{D}_{\mathfrak{w}_{k}} \mathfrak{t}=\mathbf{D}_{\mathfrak{w}} \mathfrak{t}$. Observe first that the sequence $\left(n \mathfrak{w}_{k}\right)_{k \in \mathbb{N}}$ is also monotonically nondecreasing, $\sup _{k \in \mathbb{N}} n \mathfrak{w}_{k}=n \mathfrak{w}$, and $n \mathfrak{w} \leq n \alpha \mathfrak{t}$. Consequently, we can take the supremum in $k$ :

$$
\sup _{k \in \mathbb{N}} \mathbf{D}_{\mathfrak{w}_{k}} \mathfrak{t}=\sup _{k \in \mathbb{N}}\left(\sup _{n \in \mathbb{N}} \mathfrak{t}: n \mathfrak{w}_{k}\right) \geq \mathfrak{t}: n \mathfrak{w}_{k} \Rightarrow \sup _{k \in \mathbb{N}} \mathbf{D}_{\mathfrak{w}_{k}} \mathfrak{t} \geq \mathfrak{t}: n \mathfrak{w} .
$$

This gives immediately that $\sup _{k \in \mathbb{N}} \mathbf{D}_{\mathfrak{w}_{k}} \mathfrak{t} \geq \mathbf{D}_{\mathfrak{w}} \mathfrak{t}$. Since the inequality $\mathfrak{w}_{k} \leq \mathfrak{w}$ implies

$$
\mathbf{D}_{\mathfrak{w}_{k}} \mathfrak{t} \leq \mathbf{D}_{\mathfrak{w}} \mathfrak{t}
$$

for all $k \in \mathbb{N}$, the proof is complete.

Corollary 1 Let $\mathfrak{t}$ and $\mathfrak{w}$ be nonnegative sesquilinear forms on the complex linear space $\mathfrak{D}$. Then

$$
\mathbf{D}_{\mathfrak{w}} \mathfrak{t}=\mathbf{D}_{\mathbf{D}_{\mathfrak{w}}} \mathfrak{t}
$$

i.e., $\mathbf{D}_{\mathfrak{w}} \mathfrak{t}$ is a $\mathrm{t}$-quasi-unit for all $\mathfrak{w}$. 
Acknowledgments The authors wish to express their gratitude to Zsigmond Tarcsay for pointing out the error.

\section{References}

1. Eriksson, S.L., Leutwiler, H.: A potential theoretic approach to parallel addition. Math. Ann. 274, 301-317 (1986)

2. Hassi, S., Sebestyén, Z., de Snoo, H.: Lebesgue type decompositions for nonnegative forms. J. Funct. Anal. 257(12), 3858-3894 (2009)

3. Sebestyén, Z., Titkos, T.: Parallel subtraction of nonnegative forms. Acta Math. Hung. 136(4), 252-269 (2012) 